\title{
Síndrome de Down, envejecimiento y lenguaje: consideraciones para el abordaje fonoaudiológico
}

\author{
Down syndrome, aging and language: \\ considerations for the speech-language therapy
}

\section{Maximiliano Gaete E. Fonoaudiólogo Corporación COOCENDE Santiago de Chile}

\section{Autor correspondiente: Maximiliano Gaete E. \\ Santiago - Chile Correo-e: maximiliano.gaete@gmail.com}

Recibido: 29/05/2017 Aceptado: 20/09/2017

\section{RESUMEN}

El envejecimiento de las personas con Síndrome de Down ha generado un interés creciente para las disciplinas afines al trabajo terapéutico con personas en situación de discapacidad intelectual. La fonoaudiología ha contribuido con un gran cuerpo de investigaciones al establecer procedimientos de evaluación e intervención en las habilidades comunicativas, lingüísticas y cognitivas de las personas con Síndrome de Down principalmente durante etapas tempranas y en la adolescencia. Sin embargo, el progresivo incremento de la esperanza de vida en esta población, por los avances médicos, terapéuticos y en los servicios sociales, supone nuevos desafíos para el ejercicio clínico. En particular, ello se evidencia si se considera que presentan un envejecimiento prematuro, acelerado y donde ciertas condiciones neurobiológicas predisponen la aparición temprana de procesos neurodegenerativos. En este artículo, se revisan las principales características del lenguaje de los adultos con Síndrome de Down, considerando las especificidades durante el proceso de envejecimiento y su relación con la Enfermedad de Alzheimer. Además, se describen las características de instrumentos de evaluación neuropsicológica que contemplan la valorización del lenguaje en la etapa adulta y se señalan estrategias de abordaje fonoaudiológico aplicables al trabajo de las capacidades del lenguaje de adultos con Síndrome de Down. Finalmente, se comenta la escasez de investigaciones sobre la caracterización e intervención de los dominios lingüísticos y comunicativos en la población adulta con Síndrome de Down. Se destaca también la necesidad de realizar estudios locales desde el ámbito fonoaudiológico dado el cambio demográfico de las personas en situación de discapacidad intelectual en Chile.

Palabras clave: Síndrome de Down, envejecimiento, lenguaje, enfermedad de alzheimer, fonoaudiología, intervención.

\begin{abstract}
The aging phenomenon of persons with Down Syndrome has gained increasing interest among disciplines related to therapeutic work with people with intellectual disability. Speech and language therapy has contributed with a large research body aimed at establishing evaluation and intervention procedures on communications and linguistic and cognitive skills of persons with Down Syndrome, mainly in early ages and teenagers. However, the life expectancy of people with Down Syndrome has shown a gradual increase, due to the progress made in the medical and therapeutic scope and in social services, which represent new challenges for clinical exercise, considering the course of a premature and accelerated aging, where certain neurobiological conditions influence the early manifestation of neurodegenerative processes. The present paper reviews the main language characteristics of adults with Down Syndrome, considering the specificities in the aging process and its relationship with Alzheimer's disease. Furthermore, it describes the characteristics of instruments of neuropsychological assessment that includes the valorization of the language in the adult stage, and it points out strategies of the speech and language therapy approach that are applicable to the work of language capabilities of adults with Down Syndrome. Finally, the paper addresses a shortage of research dealing with the characterization and intervention of linguistic and communicative fields among the adult population with Down Syndrome, highlighting the need to develop local studies from the speech and language therapy field given the demographic change of people with intellectual disabilities in Chile.
\end{abstract}

Keywords: Down syndrome, aging, language, Alzheimer's disease, speech-language 


\section{Introducción}

El cambio radical en la expectativa de vida media de las personas con Síndrome de Down (SD), quienes actualmente alcanzan edades superiores a los 60 años, incluso con casos que superan los 70 años, se debe a los grandes avances en el plano sanitario y en la actualización de los servicios sociales que brindan apoyos para las personas en situación de discapacidad intelectual (Bittles \& Glasson, 2004; Farriols, 2012). La incorporación gradual de este colectivo en programas de salud con atención multidisciplinar, en sistemas educativos inclusivos, en contextos de inserción laboral y programas de vida independiente ha repercutido positivamente en su estilo y calidad de vida (Berzosa, 2013; World Health Organization, 2000).

En la actualidad, se dispone de una cantidad importante de evidencia científica sobre la condición de salud general en la etapa adulta y avanzada del ciclo vital de las personas con SD. En los hallazgos asociados al proceso de envejecimiento se describen, principalmente, cambios prematuros y acelerados en relación a su edad, a nivel físico, cognitivo y en su desempeño funcional (Farriols, 2012). Así también se reporta una alta prevalencia de condiciones de salud, frecuentemente no detectadas o pobremente intervenidas, tales como pérdidas visuales y de agudeza auditiva (adicionales a las preexistentes), obesidad, problemas gastrointestinales, hipotiroidismo, osteorporosis, apnea del sueño, dificultades cognitivas y conductuales, depresión, entre otras (Haveman et al., 2010).

La enfermedad de Alzheimer, incluida en los Trastornos Neurocognitivos Mayores según el actual marco conceptual del DSM-V, lidera el listado de procesos patológicos de posible presentación en la etapa adulta del ciclo vital en el SD. Esta enfermedad se constituye como un cuadro clínico de tipo degenerativo y progresivo que se expresa, principalmente, por un descenso en los niveles de desempeño en las actividades de la vida diaria (McKhann et al., 2011) y con alteraciones en la esfera conductual y la función mnésica (Chapman \& Hesketh, 2000; Flórez, 2010; Holland, Hon, Huppert, Stevens \& Watson, 1998; Roberts, Price \& Malkin, 2007; Rondal y Ling, 2006).

Sin embargo, a pesar de que en la actualidad las personas con SD alcanzan etapas más avanzadas en su ciclo vital y se evidencia la necesidad de brindar apoyos multidisciplinares en edades adultas, el proceso de envejecimiento de esta población representa un fenómeno reciente y relativamente desconocido a la luz del contexto social. Esta situación se refleja en la oferta de programas sociosanitarios que, principalmente, van dirigidos a la estimulación temprana, al apoyo escolar y al inicio de la etapa laboral, sin considerar apoyos sistemáticos específicos para el colectivo de mayor edad (Madrigal, 2006). La situación es bastante similar en el ámbito de la investigación e intervención de las habilidades lingüísticas, comunicativas y cognitivas de las personas con SD. En este caso los principales focos de acción se centran en etapas tempranas y juveniles del ciclo vital, mientras que las experiencias de abordaje en etapas adultas son aún muy escasas (lacono, Torr \& Wong, 2010).

Dada la necesidad de visibilizar el fenómeno del envejecimiento de las personas con SD y de favorecer el acceso a programas específicos acorde a 
sus necesidades de apoyo, en este trabajo se presenta una revisión de las principales características del lenguaje durante la adultez, su relación con la presencia de procesos neurodegenerativos como la Enfermedad de Alzheimer, la descripción de instrumentos de evaluación neuropsicológica para adultos en situación de discapacidad intelectual que contemplan su valoración y, por último, se señalan ciertas directrices específicas de intervención desde el ámbito fonoaudiológico.

\section{Desarrollo}

\section{El fenómeno lingüístico en la etapa adulta}

Durante todo el ciclo vital, las personas con SD enfrentan enormes retos respecto al desarrollo del lenguaje y sus habilidades comunicativas, dominios que tienen un estrecho vínculo con el desempeño académico, la autonomía, las actividades laborales y su aporte, y la participación en el contexto social, entre otros (Kent \& Vorperian, 2013). Las personas con SD que alcanzan la adultez se desempeñan en una gran cantidad de contextos con diversos niveles de autonomía y competencias resultantes de su formación y experiencias a lo largo del ciclo vital. En este proceso de desarrollo, el lenguaje se constituye como una dimensión fundamental.

\subsection{Características generales del lenguaje en el SD}

Pese a las diferencias individuales y la heterogeneidad del perfil de desarrollo de las personas con SD, existe consenso al caracterizar su lenguaje en la existencia de asincronía entre las capacidades lingüísticas expresivas y comprensivas, dado que se observa un mejor desempeño en esta última dimensión (Rondal y Ling, 2006). Diversos autores señalan también ciertas características más específicas que se exponen a continuación:

a) La pragmática es un área de relativa fortaleza en las personas con SD (Abbeduto, Warren \& Conners, 2007). Así, es posible observar en ellas un repertorio amplio de actos comunicativos intencionales verbales y/o no verbales (Rondal y Comblain, 1996).

b) Los aspectos léxicos y semánticos presentan un desempeño superior en comparación con el manejo de la sintaxis y de la morfología (Chapman \& Hesketh, 2000; Roberts et al., 2007). Las principales deficiencias en estas últimas áreas se relacionan con dificultades importantes como la ausencia frecuente de conectores discursivos, inestabilidad en el manejo de morfemas gramaticales y un escaso uso de verbos y flexiones verbales (Hesketh \& Chapman, 1998; Martin, Klusek, Estigarribia \& Roberts, 2009).

c) Las dificultades fonológicas más importantes que se observan son omisiones de estructuras silábicas a nivel de palabra o asimilaciones (Kumin \& Adams, 2000).

Las características del lenguaje antes mencionadas se manifiestan de modo simultáneo con alteraciones de la voz, prosodia, fluidez y articulación del habla que afectan directamente la inteligibilidad de los enunciados (Kumin \& Adams, 2000; Kent \& Vorperian, 2013).

El lenguaje expresivo y comprensivo continúa su desarrollo durante la adolescencia y la edad adulta manteniendo la asincronía ya descrita entre ambos dominios (Vicari, Caselli \& Tonucci, 2000). En este desarrollo, se observan cambios principalmente en el 
incremento a nivel de repertorio y variabilidad léxica. Este incremento se mantiene con cierta estabilidad hasta los 35 años (Carr, 2003), constituyéndose como un fenómeno muy asociado a la participación del joven y adulto con SD en una mayor variedad de contextos y experiencias significativas de vida (Facon, Grubar \& Gardez, 1998).

Anteriormente se planteaba que las personas con SD alcanzaban una "meseta" en su desarrollo sintáctico durante los primeros años de adolescencia, sin embargo, en estudios más recientes se evidencia que, tanto en adolescentes como en adultos jóvenes, el desarrollo de esta dimensión del lenguaje continúa hasta la adultez (Chapman, Hesketh \& Kistler, 2002; Miles, Chapman \& Sindberg, 2006).

\subsection{Envejecimiento y desempeño lingüístico}

De igual modo que la población en general, durante el proceso de envejecimiento las personas con SD experimentan un descenso en sus capacidades cognitivas que se expresa de manera bastante heterogénea. Así, evidencian dificultades para retener información nueva o no familiar, una menor capacidad para recuperar información ya registrada en la memoria a largo plazo, una disminución tanto en las habilidades lingüísticas globales como en las funciones ejecutivas y un enlentecimiento en los tiempos de reacción frente a los estímulos del medio (Bigby, 2004; Esteba-Castillo, Ribas, Baró i Dilmé \& Novell, 2006; Signo, Bruna, Guerra-Balic, Fernández y Canals, 2016). Estos descensos podrían expresarse en torno a los 40-45 años dado un envejecimiento prematuro y acelerado (Zigman \& Lott, 2007).
En relación a los cambios en las habilidades verbales de los adultos con SD, en estudios realizados con adultos de edades entre los 19 y 64 años, se observó que el $70 \%$ de los participantes mayores de 38 años tuvo dificultades en tareas de nominación y tendencia a los circunloquios, desempeño observado principalmente en aquellas palabras menos habituales en su repertorio diario (Ribes \& Sanuy, 2001). Se ha señalado también que a partir de los 40 años se observa un descenso en el lenguaje receptivo (Cooper \& Collacott, 1995).

Por otra parte, en adultos con SD que no manifestaban sintomatología asociada a un proceso neurodegenerativo patológico, se ha observado que las habilidades lingüísticas descendidas fueron especialmente la comprensión verbal de instrucciones, la nominación de objetos y la fluidez verbal (Ghezzo et al., 2014). Recientemente, se han reportado antecedentes sobre cambios significativos a nivel neuropsicológico en el dominio mnésico, lingüístico y en el estado cognitivo general de adultos con SD mayores de 38 años, en los cuales se observó un descenso en el desempeño tanto en tareas de memoria y reconocimiento de imágenes como en aquellas de nominación viso-verbal (Signo, 2016).

\subsection{Enfermedad de Alzheimer y su impacto en el lenguaje}

En la etapa adulta del ciclo vital de la persona con $\mathrm{SD}$, emerge un fenómeno crítico en términos lingüísticos. Este fenómeno tiene relación directa, por una parte, con el proceso de envejecimiento prematuro y acelerado con cambios en la esfera cognitiva $y$, por otra, con lo referido al limitado tiempo entre la consolidación de las habilidades del 
lenguaje desarrolladas en la adultez y el precoz inicio del declive cognitivo asociado a la Enfermedad de Alzheimer (EA) (lacono et al., 2010). Al respecto, se ha señalado que a partir de los 35 años los adultos con SD son más proclives a desarrollar este Trastorno Neurocognitivo Mayor (Glasson, Dye y Bittles, 2014.)

El desarrollo de la EA en las personas con SD se asocia a condiciones particulares a nivel neurobiológico que predisponen la acumulación intraneuronal de $\beta$-amiloide y la formación de ovillos neurofibrilares (Flórez, Garvía y Fernández-Olaria, 2015; Lott, 2012). En la fase inicial de presentación de la EA en personas con SD, comienzan a observarse síntomas de corteza prefrontal tales como una comunicación social deficiente, apatía, falta de cooperación, indiferencia y depresión (Ball et al., 2006). Al progresar esta sintomatología se advierten conjuntamente descensos en el desempeño adaptativo y funcional relacionados con los declives a nivel mnésico, comunicativo-lingüístico, en las funciones ejecutivas, y la presencia de rasgos apráxicos (Esteba-Castillo et al., 2006). Ya en una fase avanzada de la EA, se observan dificultades significativas en la memoria y el desplazamiento, desorientación en el hogar y espacios cotidianos, dificultades deglutorias y conductas agresivas, entre otros (para una revisión en detalle, ver National Down Syndrome Society, 2013).

El impacto en el desempeño lingüístico del adulto con SD que desarrolla la EA se expresa en los siguientes aspectos (Rondal \& Edwards,1997):

I. Un enlentecimiento en el procesamiento del lenguaje comprensivo y expresivo.

II. Dificultades adicionales a las deficiencias sintácticas previas en el seguimiento de instrucciones y en la extracción de información del discurso oral.

III. Una disminución en la fluidez verbal y en la recuperación de nombres comunes y propios.

Este perfil lingüístico se caracteriza por un deterioro variable, pero significativo, entre cada individuo (Rondal \& Comblain, 1996). Hallazgos posteriores señalan descensos tanto en el léxico receptivo como en las habilidades pragmáticas reportados con la progresión de sintomatología asociada a la EA (Nelson, Orme, Osann \& Lott, 2001).

Existe un cuerpo de investigaciones que ha dedicado esfuerzos valiosos en describir los cambios en las habilidades lingüísticas de las personas con SD asociados con la edad y particularmente en el proceso de envejecimiento o desarrollo de la EA. Sin embargo, se ha destacado la necesidad de contar con un mayor número de estudios y evidencia actualizada sobre los perfiles y caracterización de los dominios cognitivos y lingüísticos a raíz de la variabilidad de los déficits encontrados (lacono et al., 2010; Martin et al., 2009).

\section{Abordaje fonoaudiológico durante la adultez}

El reconocimiento del rol fundamental del fonoaudiólogo en los procesos terapéuticos de los dominios del lenguaje, la comunicación y cognición en la población infanto-juvenil y adulta con SD cobra cada vez mayor fuerza. Ello surge de los avances en investigaciones que sustentan el actuar disciplinar y su impacto en la calidad de vida de esta población (Berzosa, 2013; Jenkins, 2003; Jokinen et al., 2013; Perera, Flórez \& Rondal, 2013). Al respecto, se ha señalado que las personas con SD se benefician al 
participar de terapias fonoaudiológicas orientadas a desarrollar las habilidades del lenguaje y habla durante todo el ciclo vital, incluyendo su mantención en edades avanzadas (Buckley, 1999).

\subsection{Evaluación lingüística y cognitiva del adulto con} SD

En la actualidad existen limitaciones para establecer un proceso evaluativo de las capacidades lingüísticas de la población adulta con SD. Lo anterior se debe a la escasez de instrumentos específicos orientados al establecimiento de perfiles neuropsicológicos y del desempeño en las dimensiones del lenguaje en la etapa adulta del ciclo vital de las personas en situación de discapacidad intelectual.

A pesar de ello, uno de los instrumentos que se vislumbra como una alternativa concreta para este proceso es la prueba "Cambridge Examination for Mental Disorders of Older People with Down's Syndrome and others with intellectual disabilities", CAMDEX.DS (Ball, Holland, Huppert, Treppner \& Dodd, 2006). Esta prueba ha sido adaptada y validada para la lengua española (Esteba-Castillo et al., 2013). El CAMDEX-DS es un instrumento de evaluación de tipo cribado cuya finalidad es facilitar el diagnóstico de demencia y otros trastornos mentales en la población con discapacidad intelectual mayor de 30 años. A través de una entrevista estructurada a un informante, quien proporciona información sobre el estado actual del usuario y de su historia médica, se obtiene una descripción de las características cognitivas generales del adulto y sobre el progreso de las intervenciones multidisciplinares. Por otra parte, el CAMDEX-DS cuenta con la batería neuropsicológica CAMCOG-DS para la valoración de las funciones cognitivas. En ella, se presenta una evaluación directa al adulto que contempla, entre otros dominios cognitivos, ítems de evaluación para lenguaje comprensivo (incluyendo tareas de seguimiento de instrucciones verbales y comprensión lectora) y expresivo (con tareas de denominación, fluidez verbal, definición de conceptos y repetición de enunciados). Los puntajes obtenidos se contrastan con los criterios diagnósticos para demencia del DSM-IV y CIE-10.

En una línea muy similar, recientemente se publicó un estudio sobre la aplicación del "Test de Barcelona para Discapacidad Intelectual (TB-DI)" con población adulta, instrumento de evaluación neuropsicológica que contempla ocho dominios cognitivos: Orientación, Atención, Memoria de Trabajo, Lenguaje, Praxis, Memoria, Funciones Ejecutivas y Visuoconstrucción. Este test posee buenas propiedades psicométricas así como una validez y fiabilidad adecuadas. No obstante, los autores señalan la importancia de la experiencia clínica del profesional con población adulta en situación de discapacidad intelectual cuando se establece el proceso de valoración neuropsicológica con esta prueba (Esteba-Castillo et al., 2017).

La "Guía de Identificación de Indicios de Envejecimiento y Orientaciones para la determinación de Apoyos" es otro instrumento que permite reconocer y valorar los cambios producidos en la persona durante la etapa adulta de su ciclo vital. Evalúa el estado actual y la evolución de las habilidades relacionadas con la comunicación, el lenguaje, los procesos cognitivos básicos, la coordinación, motricidad y su desarrollo socio- 
emocional. Su aplicación permite vislumbrar cuándo se produjeron los cambios que están impactando en su calidad de vida, a qué se deben y cuál es la influencia del proceso de envejecimiento en ellos. Esta información se obtiene con la finalidad de establecer pautas y directrices de apoyo concreto que requieren los adultos para mejorar sus capacidades funcionales y mantener su autonomía (FEAPS Madrid, 2006).

\subsection{Establecimiento de un marco de acción}

Es necesario planificar intervenciones basadas en los contextos y necesidades comunicativas que van presentando las personas con SD al recorrer la etapa adulta del ciclo vital (Rondal, 2003; Jenkins, 2003). Los programas de intervención deben enmarcarse y sustentarse en modelos que consideren, por una parte, la evidencia disponible para el abordaje específico en personas con SD en los dominios lingüísticos y cognitivos y, por otra, en lineamientos que rescaten la base empírica sobre estrategias terapéuticas aplicadas en población adulta que desarrolla procesos neurodegenerativos. Este encuadre de intervención se ve enriquecido con la denominada Planificación Centrada en la Persona $(P C P)$, constructo que actualmente lidera la planificación y establecimiento de los sistemas de apoyo individual basado en el reconocimiento de las actividades, experiencias, preferencias y decisiones personales de los adultos en situación de discapacidad intelectual (Johnson, Douglas, Bigby \& lacono, 2009; Mata \& Carratalá, 2007).

\subsection{Directrices de abordaje lingüístico}

Los objetivos de intervención desde el prisma fonoaudiológico se desprenden del análisis de las necesidades de apoyo individuales y buscan incrementar o mantener las habilidades en los dominios comunicativo-lingüísticos del adulto con SD a través de actividades altamente significativas, acorde a sus actividades y demandas funcionales, favoreciendo su calidad de vida (Hopper, Douglas \& Khayum, 2015; Pelatti, 2015; Perera et al., 2013). A continuación, se presentan ciertos principios generales y directrices aplicables a la intervención fonoaudiológica del lenguaje con adultos con SD:

- La intervención requiere del planteamiento de un programa estructurado, sistemático, que incluya objetivos concretos, alcanzables y vinculados a los intereses y necesidades comunicativas del adulto con SD (Jenkins, 2003).

- La planificación de las actividades necesita considerar las fortalezas del procesamiento visual para la retención de información (Buckley, 1999; Jenkins, 2003; Rondal, 2003).

- En el trabajo con adultos, es necesario realizar adecuaciones de los referentes de intervención, procurando que el material de las actividades tenga imágenes de situaciones reales bien definidas, de tamaño grande y manipulable (Lodeiro et al., 2002).

- La intervención debe buscar incrementar los periodos de comunicación a través de actividades que demanden bastante interacción social, generando mayores oportunidades de desarrollar o mantener las habilidades léxicas y morfosintácticas (Buckley, 1999).

- Se debe reforzar y mantener las habilidades lingüísticas y competencias sociales mediante actividades de interacción que impliquen la 
expresión verbal utilizando tópicos asociados a sus actividades diarias, intereses personales $y$ proyecciones acordes a la etapa adulta de su ciclo vital, permitiendo disfrutar socialmente del intercambio comunicativo (Buckley, 1999; Schelstraete \& Lezcano, 2016).

- Es fundamental desarrollar actividades sistemáticas con el objetivo de mantener o incrementar el repertorio léxico funcional y las relaciones semánticas a través del uso de organizadores visuales de palabras, el establecimiento de redes semánticas o el uso de narraciones, considerando las exigencias comunicativas del adulto, sus contextos de participación y experiencias significativas (Pelatti, 2015; Rondal, 2003).

- En las actividades se debe incluir un input multisensorial a través de material visual, auditivo, olfativo y táctil (Buckley, 1999).

- En el abordaje con adultos que presentan mayores necesidades de apoyo comunicativo, como es el caso de personas con una discapacidad intelectual severa o que presentan sintomatología asociada a la EA, se debe favorecer la inclusión de Sistemas de Comunicación Alternativos-Aumentativos (SCAA) con el objetivo de entregar un soporte visual tanto en la planificación de las Actividades de la Vida Diaria como una alternativa de apoyo comunicativo a nivel expresivo-receptivo (Johnson et al., 2009).

Otros constructos y metodologías de abordaje son las denominadas Intervenciones Directas e Indirectas, las cuales han mostrado positivos resultados a nivel cognitivo y comunicativo en, por ejemplo, personas que desarrollan un Trastorno Neurocognitivo Mayor (Hopper et al., 2013). Las Intervenciones Directas son aquellas orientadas a mantener las habilidades cognitivo-lingüísticas residuales de la persona, apoyando sus rutinas diarias y previniendo un incremento en los niveles de discapacidad comunicativa (Clark, 1995) y en las que se incluyen el uso de estrategias específicas, tales como la recuperación espaciada, el desvanecimiento de claves y el aprendizaje sin errores (Hopper et al., 2015).

Las Intervenciones Indirectas, por su parte, están enfocadas en incrementar significativamente el bienestar de la persona mediante adecuaciones en los contextos físico y psicosocial, promover su participación positiva en rutinas en ambientes de cuidado y favorecer instancias de capacitación a cuidadores y a su red de apoyo en técnicas de comunicación efectiva (Clark, 1995). Las principales estrategias a implementar se relacionan con establecer dinámicas conversacionales en lugares iluminados, reducir el ruido de fondo y donde el interlocutor/cuidador, ya capacitado en técnicas de manejo discursivo, se comunique utilizando expresiones verbales más breves y simples, hablando lentamente, con una intensidad de voz un poco más elevada, acompañando los enunciados con gestos y material de apoyo para contextualizar (como fotografías o imágenes) y otorgando al adulto con SD tiempo adicional para comprender y expresarse (Buckley, 1999; Johnson et al., 2009; Hopper, 2001; Rondal, 2003).

2.4. Proyecciones del ámbito fonoaudiológico en el trabajo con adultos con SD 
Si bien la actuación fonoaudiológica ha tenido un importante impacto en el ámbito del desarrollo de las personas con SD durante etapas tempranas y juveniles del ciclo vital, es necesario contar con un cuerpo más robusto de investigaciones que sustenten la práctica disciplinar en los procesos de evaluación y de intervención en los dominios lingüísticos y comunicativos en población adulta con SD. Se ha propuesto que las investigaciones destinadas al establecimiento de los perfiles comunicativo-lingüísticos de este grupo cuenten con muestras más amplias, donde se comparen los desempeños entre aquellos individuos que presentan una EA y aquellos que no la han desarrollado y que en los estudios participen investigadores formados en disciplinas afines a los Trastornos de la comunicación que cuenten con experiencia clínica en el trabajo con adultos con SD (lacono et al., 2010).

El contexto social y las actividades en que participan las personas con discapacidad intelectual serán determinantes en su experiencia de envejecimiento (Oliver, Adams \& Kalsy, 2008). Por lo tanto, es necesario que el fonoaudiólogo contemple el desarrollo e implementación de programas de intervención anticipados para adultos con SD desde los 30 años. Lo anterior permitiría fortalecer el proceso de envejecimiento saludable a través del reforzamiento y mantención de sus habilidades comunicativas, cognitivas y funcionales. Además, transversalmente, es recomendable promover programas de apoyo junto a un equipo transdisciplinario que estén dirigidos a favorecer su participación y desempeño en las áreas laborales, actividades físicas, socialización y un estilo de vida activo (Esteba-Castillo et al., 2006; Flórez et al., 2015; Lin et al., 2014; Mascarenhas, Rufino, Bottino \& Correa, 2015).

$\mathrm{Si}$ bien es cierto aquí se ha presentado una revisión general sobre los antecedentes disponibles para el abordaje fonoaudiológico comunicativolingüístico de la población adulta con SD, la idea de realizar futuras investigaciones locales que caractericen el desempeño o perfilen sus necesidades de apoyo en estos dominios durante el proceso de envejecimiento no debe ser desestimada, reconociendo que en el colectivo de personas en situación de discapacidad intelectual en Chile también se comienza a observar un cambio demográfico (para una revisión en detalle del fenómeno en términos estadísticos, ver Observatorio de Derechos Humanos de las Personas con Discapacidad Mental, 2014).

\section{Conclusiones}

Como se señala en este artículo, la evidencia disponible sobre el abordaje lingüístico y comunicativo durante la etapa adulta del ciclo vital de las personas con SD aún es limitada. Pese a ello, de los estudios consultados emergen consideraciones sustanciales que permiten establecer un planteamiento fonoaudiológico para esta población. Al respecto, en primer lugar, es recomendable que el abordaje terapéutico contemple las especificidades neurobiológicas (por ejemplo, probable presentación precoz de procesos neurodegenerativos patológicos) y el perfil prematuro y acelerado del proceso de envejecimiento incluyendo propuestas de 
intervención anticipadas dentro del marco de programas de apoyo transdisciplinario a partir de los 30 años. En segundo lugar, es conveniente cautelar que los objetivos de la intervención fonoaudiológica sean congruentes con las necesidades de apoyo lingüístico y cognitivo determinadas en la Planificación Centrada en la Persona para la mantención del bienestar, autodeterminación y calidad de vida del adulto. Finalmente, es recomendable que la línea de intervención de los dominios lingüístico y comunicativo contemple actividades sistemáticas $\mathrm{y}$ altamente significativas para el adulto con SD basadas en metodologías y estrategias que posean respaldo empírico.

Sin duda el fenómeno del envejecimiento de las personas con SD representa un importante desafío para las disciplinas afines al trabajo en el área de la discapacidad intelectual considerando el incremento de la esperanza de vida y la urgente necesidad de visibilizar y profundizar los conocimientos sobre las condiciones de salud asociadas a etapas avanzadas del ciclo vital. En este escenario, la fonoaudiología se vislumbra como una disciplina fundamental en el establecimiento de apoyos a nivel lingüístico, comunicativo y cognitivo de la población adulta con SD, proyección que debe estar asociada a la construcción previa de un campo teórico y empírico robusto que sustente con evidencia actualizada los procedimientos de evaluación e intervención reconociendo, conjuntamente, las características y particularidades locales del proceso de envejecimiento que determinan las actuales necesidades de apoyo de este grupo.

\section{Referencias}

Abbeduto, L., Warren, S. \& Conners, F. (2007). Language development in Down syndrome: from the prelinguistic period to the acquisition of literacy. Mental Retardation and Developmental Disabilities Research Reviews, 13(3), 247-261. doi:10.1002/mrdd.20158

Ball, S., Holland, A., Huppert, F., Treppner, P. \& Dodd K. (2006). Cambridge Examination for Mental Disorders of Older People with Down's Syndrome and others with intellectual disabilities, Cambridge, Cambridge University Press.

Ball, S., Holland, A., Hon, J., Huppert, F., \& Watson, P. (2006). Personality and behavior changes mark the early stages of Alzheimer's disease in adults with Down syndrome: Findings from a prospective population-based study. International Journal of Geriatric Psychiatry, 21(7): 661-673.

Berzosa, G. (dir). (2013). Las personas con síndrome de Down y sus familias ante el proceso de envejecimiento. Madrid: Real Patronato sobre Discapacidad, Down España.

Bigby, C. (2004). Aging with a lifelong disability: Policy, program and practice issues for professionals. London: Jessica Kingsley.

Bittles, A. \& Glasson, E. (2004). Clinical, social, and ethical implications of changing life expectancy in Down syndrome. Developmental Medicine \& Child Neurology, 46, 282-286.

Buckley, S. (1999). Improving the speech and language skills of children and teenagers with Down Syndrome. Down Syndrome News and Update, 3(1), 111- 128.

Carr, J. (2003). Patterns of Ageing in 30-to-35 year olds with Down's Syndrome. Journal of Applied Research in Intellectual Disabilities, 16, 29-40.

Chapman, R. \& Hesketh, L. (2000). Behavioral phenotype of individuals with Down Syndrome. Mental Retardation and Developmental Disabilities Research Reviews, 6(2), 84-95.

Chapman, R., Hesketh, L. \& Kistler, D. (2002). Predicting longitudinal change in language production and comprehension in individuals with Down syndrome: hierarchical linear modeling. Journal of Speech, Language, 
and Hearing Research, 45(5), 902-915.

Clark, L. (1995). Interventions for persons with Alzheimer's disease: Strategies for maintaining and enhancing communicative success. Topics in Language Disorders, 15(2), 47-65.

Cooper, S. \& Collacott, R. (1995). The effect of age on language in people with Down's syndrome. Journal of Intellectual Disability Research, 39, 197-200.

Esteba-Castillo, S., Dalmau-Bueno, A., Ribas-Vida, N., VilaAlsina, M., Novell-Alsina, R. \& García-Alba, J. (2013). Adaptación y validación del Cambridge Examination for Mental Disorders of Older People with Down's Syndrome and Others with Intellectual Disabilities (CAMDEX-DS) en población española con discapacidad intelectual. Revista de Neurología, 57(8), 337-346.

Esteba-Castillo, S., Peña-Casanova, J., García-Alba, J., Castellanos, M., Torrents-Rodas, D., Rodríguez, E. \& NovellAlsina, R. (2017). Test de Barcelona para discapacidad intelectual: un nuevo instrumento para la valoración neuropsicológica clínica de adultos con discapacidad intelectual. Revista de Neurología, 64(10), 433-444.

Esteba-Castillo, S., Ribas, N., Baró i Dilmé, M., \& Novell, R. (2006). Envejecimiento saludable en personas con síndrome de Down y demencia: necesidad de promover programas de formación y soporte a los usuarios, familias y entidades. Revista Médica internacional sobre el Síndrome de Down, 10(2), 25-29.

Farriols, C. (2012). Aspectos específicos del envejecimiento en el Síndrome de Down. Revista Médica internacional sobre el Síndrome de Down, 16(1), 3-10.

Facon, B., Grubar, J., \& Gardez, C. (1998). Chronological age and receptive vocabulary of persons with Down Syndrome. Psychological Reports, 82, 723-726.

FEAPS Madrid. (2006). Las personas con necesidades de apoyo generalizado: guía de identificación de indicios de envejecimiento y orientaciones para la determinación de apoyos. Cuadernos de Atención de Día. FEAPS Madrid.

Flórez, J. (2010). Enfermedad de Alzheimer y síndrome de Down. Revista síndrome de Down, 27, 63-76. Recuperado de

https://www.downciclopedia.org/envejecimiento1/enferm edad-de-alzheimer-y-sindrome-de-down
Flórez, J., Garvía, B., \& Fernández-Olaria, R. (2015). Síndrome de Down: Neurobiología, Neuropsicología, Salud Mental. Madrid, España: Fundación Iberoamericana Down21 y CEPE editorial.

Glasson, E., Dye, D. \& Bittles, A. (2014). The triple challenges associated with age-relates comorbidities in Down syndrome. Journal of Intellectual Disability Research, 58, 393-398.

Ghezzo, A., Salvioli, S., Solimando, M., Palmieri, A., Chiostergi, C., Scurti, M., ... Franceschi, C. (2014). AgeRelated Changes of Adaptive and Neuropsychological Features in Persons with Down Syndrome. PLOS ONE 9(11), 1-21. doi:10.1371/journal.pone.0113111

Haveman, M., Heller, T., Lee, L., Maaskant, M., Shooshtari, S., \& Strydom, A. (2010). Major health risks in aging persons with intellectual disabilities: an overview of recent studies. Journal of Policy and Practice in Intellectual Disabilities, 7(1), 59-69.

Hesketh, L. \& Chapman, S. (1998). Verb Use by Individuals with Down syndrome. American Journal on Mental Retardation, 103(3), 288-304.

Holland, A., Hon, J., Huppert, F., Stevens, F. \& Watson, P. (1998). Population-based study of the prevalence and presentation of dementia in adults with Down syndrome. British Journal of Psychiatry, 172, 493-498.

Hopper, T. (2001). Indirect Interventions to Facilitate Communication in Alzheimer's Disease. Seminars in Speech and Language, 22(4), 305-315.

Hopper, T., Bourgeois, M., Pimentel., Dean Qualls, C., Hickey, E., Frymark, T. \& Schooling, T. (2013). An EvidenceBased Systematic Review on Cognitive Interventions for Individuals with Dementia. American Journal of SpeechLanguage Pathology, 22, 126-145. doi:10.1044/10580360(2012/11-0137)

Hopper, T., Douglas, N. \& Khayum, B. (2015). Direct and indirect interventions for cognitive-communications disorders of dementia. Perspectives on Neurophysiology and Neurogenic Speech and Language Disorders, 25, 142157.

lacono, T., Torr, J. \& Wong, H. (2010). Relationships amongst age, language and related skills in adults with 
Down syndrome. Research in Developmental Disabilities, 31, 568-576. doi:10.1016/j.ridd.2009.12.009

Jenkins, C. (2003). Continued language intervention with adolescents and adults with Down syndrome. En J. Rondal y S. Buckley (Eds.), Speech and Language Intervention in Down Syndrome (pp. 154-165). London, England: Whurr.

Johnson, H., Douglas, J., Bigby, C. \& lacono, T. (2009). Maximizing community inclusion through mainstream communication services for adults with severe disabilities. International Journal of Speech-Language Pathology, 11(3), 180-190.

Jokinen, N., Janicki, M.P., Keller, S.M., McCallion, P., Force, L.T., and the National Task Group on Intellectual Disabilities and Dementia Practices. (2013). Guidelines for Structuring Community Care and Supports for People With Intellectual Disabilities Affected by Dementia. Albany NY: NTGIDDP \& Center for Excellence in Aging \& Community Wellness.

Kent, R. \& Vorperian, H. (2013). Speech Impairment in Down Syndrome: A Review. Journal of Speech Language and Hearing Research, 56, 178-210. doi:10.1044/10924388(2012/12-0148)

Kumin, L. \& Adams, J. (2000). Developmental apraxia of speech and intelligibility in children with Down syndrome. Down Syndrome Quarterly, 5, 1-6.

Lin, J., Lin, L., Hsu, S., Chen, W., Lin, F., Wud, J., \& Chu, C. (2014). Are early onset aging conditions correlated to daily activity functions in youth and adults with Down syndrome? Research in Developmental Disabilities, 36, 532-536. doi:10.1016/j.ridd.2014.10.051

Lodeiro, L., Varela, N., López, A., Gandoy, M., \& Millán, J. (2002). Papel del logopeda en un centro gerontológico de estancias diurnas. Revista de Logopedia, Foniatría y Audiología, 22(1), 42-48.

Lott, I. (2012). Neurological phenotypes for Down syndrome across the life span. Progress in Brain Research, 197, 101-121. doi:10.1016/B978-0-444-54299-1.00006-6.

Madrigal, A. (2006). Marco Teórico del Envejecimiento de las Personas con Discapacidad Intelectual. Observatorio de personas Mayores, Madrid, España.

Martin, G., Klusek, J., Estigarribia, B. \& Roberts, J. (2009).
Language characteristics of individuals with Down Syndrome. Topics in Language Disorders, 29(2), 112-132.

Mascarenhas, L., Rufino, A., Bottino, C. \& Correa, E. (2015). Cognitive Rehabilitation of Dementia in Adults with Down Syndrome: A Review of Non-Pharmacological Interventions. Dementia and Geriatric Cognitive Disorders Extra, 5, 330-340. doi:10.1159/000438858

Mata, R., G. \& Carratalá, M., A. (2007). Planificación Centrada en la Persona. Experiencia de la Fundación San Francisco de Borja para Personas con Discapacidad Intelectual. FEAPS.

McKhann, G., Knopman, D., Chertkow, H., Hyman, B., Jack, C. \& Kawas, C., Phelps, C. (2011). The diagnosis of dementia due to Alzheimer's disease: Recommendations from the National Institute on Aging and the Alzheimer's Association workgroup. Alzheimers \& Dementia, 7(3), 263269. doi:10.1016/j.jalz.2011.03.005

Miles, S., Chapman, R. \& Sindberg, H. (2006). Sampling context affects MLU in the language of adolescents with Down syndrome. Journal of Speech, Language and Hearing Research, 49(2), 325-337.

National Down Syndrome Society. (2013). Aging and Down Syndrome: A Health \& Well-Being Guidebook.

Nelson, L., Orme, D., Osann, K. \& Lott, I. (2001). Neurological changes and emotional functioning in adults with Down syndrome. Journal of Intellectual Disability Research, 45, 450-456.

Observatorio de Derechos Humanos de las Personas con Discapacidad Mental. (2014). Derechos Humanos de las Personas con Discapacidad Mental: Diagnóstico de la Situación en Chile. Santiago, Chile.

Oliver, C., Adams, D. \& Kalsy, S. (2008). Ageing, dementia and people with intellectual disability. En R. Woods y L. Clare (Eds.), Handbook of the Clinical Psychology of Ageing (pp. 341-350). Wiley: Chichester.

Pelatti, C. (2015). Enhancing Oral and Written Language for Adolescents and Young Adults with Down syndrome. Seminars in Speech and Language, 36(1), 50-59. doi:10.1055/s-0034-1396446

Perera, J., Flórez, J. \& Rondal, J. (2013). Rehabilitación cognitiva de las personas con síndrome de Down: 
perspectivas multidisciplinares de normalización. Revista Síndrome de Down, 30, 106-117.

Ribes, R. \& Sanuy, J. (2001). Anomia, Demencia Alzheimer y Síndrome de Down. Revista de Logopedia, Foniatría y Audiología, 21(3), 118-123. doi:10.1016/S02144603(01)76196-7

Roberts, J., Price, J. \& Malkin, C. (2007). Language and communication development in Down Syndrome. Mental Retardardation and Developmental Disabilities Research Reviews, 13, 26-35. doi:10.1002/mrdd.20136

Rondal, J. (2003). Maintenance training in older ages. En J. Rondal y S. Buckley (Eds.), Speech and Language Intervention in Down Syndrome (pp. 154-165). London, England: Whurr.

Rondal, J. \& Comblain, A. (1996). Language in adults with Down Syndrome. Down Syndrome Research and Practice, $4(1), 3-14$.

Rondal, J. \& Edwards S. (1997). Language in mental retardation. Theory, problems, and remediation. London: Whurr.

Rondal, J. y Ling, L. (2006). Especificidad neuroconductual en el Síndrome de Down. Revista de Logopedia, Foniatría y Audiología, 26(1), 12-19.

Schelstraete, G. \& Lezcano, A. (2016). Desarrollo de la agilidad mental: El razonamiento y el pensamiento crítico a través del lenguaje oral y escrito. Revista Síndrome de Down, 33, 74-81.

Signo, S. (2016). El proceso de envejecimiento de las personas con Síndrome de Down: estudio multicéntrico para la detección de los cambios neuropsicológicos [Tesis Doctoral]. Universidad Ramón Llull. Barcelona.

Signo, S., Bruna, O., Guerra-Balic, M., Fernández, R. \& Canals, G. (2016). El proceso de envejecimiento de las personas con síndrome de Down: estudio multicéntrico para la detección de los cambios neuropsicológicos. Revista Síndrome de Down, 33, 82-93.

Vicari, S., Caselli, M. \& Tonucci, F. (2000). Asynchrony of lexical and morphosyntactic development in children with Down Syndrome. Neuropsychologia, 38, 634-644.

World Health Organization. (2000). Ageing and Intellectual Disabilities. Improving Longevity and Promoting Healthy
Ageing: Summative Report. Geneva, Switzerland: World Health Organization.

Zigman, W. \& Lott, I. (2007). Alzheimer's disease in Down syndrome: Neurobiology and risk. Mental Retardation and Developmental Disabilities Research Reviews, 13, 237-246. doi:10.1002/mrdd.20163 